\title{
Rehabilitation Program for Prosthetic Tracheojejunal Voice Production and Swallowing Function Following Circumferential Pharyngolaryngectomy and Neopharyngeal Reconstruction with a Jejunal Free Flap
}

\author{
Laura W. J. Baijens • Renée Speyer • \\ Nel Roodenburg • Frans J. M. Hilgers
}

Received: 16 November 2009/ Accepted: 16 March 2010/Published online: 3 April 2010

(C) The Author(s) 2010. This article is published with open access at Springerlink.com

\begin{abstract}
The case of a 68-year-old woman with postoperative speech and swallowing problems following a circumferential pharyngolaryngectomy and neopharyngeal reconstruction with a jejunal free flap is presented. The primary tumor was an extended papillary thyroid carcinoma (pT4N0M0). For vocal restoration, an indwelling Provox ${ }^{\circledR} 1$ voice prosthesis was inserted secondarily. The patient received speech and swallowing therapy, including digital maneuvers at the level of the proximal (cervical) part of the jejunal graft to improve speech and swallowing function. Pre- and/ or post-treatment data on speech and
\end{abstract}

L. W. J. Baijens $(\bowtie) \cdot$ R. Speyer

Department of Otorhinolaryngology, Head and Neck Surgery, Maastricht University Medical Centre, P. O. Box 5800, 6202 AZ Maastricht, The Netherlands

e-mail: laura.baijens@mumc.nl; laura.baijens4@yucom.be

R. Speyer

Comprehensive Cancer Centre West, Leiden, The Netherlands

N. Roodenburg

Department of Neurology, Maastricht University Medical

Centre, Maastricht, The Netherlands

F. J. M. Hilgers

Department of Head and Neck Surgery and Oncology, The

Netherlands Cancer Institute, Amsterdam, The Netherlands

\section{F. J. M. Hilgers}

Department of Otolaryngology-Head and Neck Surgery,

Academic Medical Centre, University of Amsterdam,

Amsterdam, The Netherlands

F. J. M. Hilgers

Institute of Phonetic Sciences/ACLC, University of Amsterdam, Amsterdam, The Netherlands swallowing function were gathered using the following assessment methods: esophageal insufflation test, Voice Handicap Index (VHI), videofluoroscopy of phonation (VFSph), digital high-speed endoscopy of jejunal vibration during voice production, fiber-optic endoscopic evaluation of swallowing (FEES), and videofluoroscopy of swallowing (VFSs). This case clearly demonstrates that even after extensive laryngopharyngectomy with jejunal free flap reconstruction, a tailored rehabilitation program can improve both voice and swallowing function, and that these results clearly can be objectified/visualized, underlining the validity of this approach.

Keywords Alaryngeal speech · Pharyngectomy · Laryngectomy $\cdot$ Surgical flaps

Rehabilitation of speech and swallowing following circumferential pharyngolaryngectomy with free jejunal flap repair remains a challenge. Tracheoesophageal puncture and insertion of a voice prosthesis has become the standard treatment protocol for voice restoration [1, 2]. Most frequently, a secondary voice restoration is performed several months following surgery [3]. Probably the jejunal convolutions and hypotonicity are major reasons leading to disordered prosthetic tracheojejunal voice production [4]. The vibration capacities of the jejunal tissue are very different compared to the pharyngoesophageal segment. Frequently, this leads to insufficient voice restoration. Ward et al. [5] describe disordered swallowing as a common complication following pharyngolaryngectomy. Stenosis of the jejunoesophageal anastomosis, absence of a functional muscular layer in the jejunal graft, and the major difference in diameter between the jejunal graft and the cervical esophagus are important causal factors of dysphagia. 
Futura et al. [3] suggest that placement of the voice prosthesis close to the jejunoesophageal anastomosis may cause scar formation resulting in deterioration of the prosthetic tracheojejunal voice production and swallowing function. In the literature, most of the studies handling prosthetic tracheojejunal voice production or swallowing function following circumferential pharyngolaryngectomy with free jejunal flap repair include rather small patient populations or do not report diagnostic imaging assessments or therapy programs for swallowing disorders or speech disorders $[6,7]$.

\section{Case Report}

A 68-year-old woman was referred to the Department of Otorhinolaryngology, Head and Neck Surgery because of speech and swallowing problems following a circumferential pharyngolaryngectomy with free jejunal repair because of an extended papillary thyroid carcinoma (pT4N0M0). Postoperatively, the patient was treated with radioactive iodide $(75,150 \mathrm{mCi})$. At this moment, 2 years after the circumferential pharyngolaryngectomy, the patient is still in complete remission.

Four months following the pharyngolaryngectomy, surgical voice restoration with an indwelling Provox ${ }^{\circledR} 1$ voice prosthesis (ATOS Medical AB, Hörby, Sweden) was carried out. Due to anatomical limitations, it was possible to insert the prosthesis only in the distal part of the jejunal graft instead of in the esophageal wall. During the first 2 months after the prosthesis was placed, the patient received standard speech rehabilitation for laryngectomees without improvement of tracheojejunal voice and swallowing function. During the next 7 months, the patient underwent training with an experienced speech-language pathologist (30 min per session three times per week). The major treatment goals of the tailored rehabilitation program (Table 1) included improvement of quality and comfort of the tracheojejunal voice function as well as improvement in the swallowing function by strengthening the musculature, using postural techniques, and using digital maneuvers (Fig. 1a-c). Speech therapy included respiratory exercises (optimization of head and body positioning) and digital maneuvers to constrict the proximal part of the jejunal graft during tracheojejunal voice production. Dysphagia treatment included tongue base exercises combined with bolus modification like changing bolus volume and/or consistency $[8,9]$. Tongue base exercises included the tongueholding maneuver and stretch and push against resistance tasks. Bolus modification was started by using smaller bolus volumes during the exercises. Gradually, bolus volumes were increased up to $10 \mathrm{cc}$ of thin liquid. Furthermore, food consistency was extended to thick liquids and pureed foods. During food intake, digital pressure was used at the level of the proximal (cervical) part of the jejunal graft to minimize jejunal convolutions by stretching the graft. A postural technique consisting of extending the neck in order to facilitate bolus transport in the jejunal graft was part of the exercise program.

In order to assess the validity of this tailored approach, speech and swallowing functions were analyzed/visualized pre- and/or post-treatment using the following assessment and imaging tools (Table 2): esophageal insufflation test, Voice Handicap Index (VHI), videofluoroscopy of phonation (VFSph), digital high-speed endoscopy of jejunal vibration during voice production, fiber-optic endoscopic evaluation of swallowing (FEES), and videofluoroscopy of swallowing (VFSs). The pretreatment videofluoroscopic studies of phonation and swallowing were used to determine the treatment strategies.

\section{Results}

\section{Tracheojejunal Voice}

At the moment of referral the tracheojejunal voice quality was wet and gravelly. The voice was produced by airflow through a jejunal constriction, independent of intestinal peristalsis [3]. Before speech therapy, an esophageal insufflation test (Blom-Singer ${ }^{\circledR}$, InHealth Technologies, Carpinteria, CA, USA) was applied to evaluate the vibration capacities of the jejunal segment by means of a transnasal catheter. This test simulates the presence of a voice prosthesis by diverting expired air from the trachea into the jejunal graft [10]. The position of the catheter tip inferior to the vibrating segment was ensured by fiber-optic endoscopy. The quality of the tracheojejunal voice did not differ between the esophageal insufflation test and the application of the Provox voice prosthesis, indicating that the function of the voice prosthesis was optimal. The VHI developed by Jacobson et al. [11] was applied as a qualityof-life questionnaire. Before treatment, the patient felt extremely handicapped by the restricted communicative capacities, whereas after treatment, the score significantly improved (from 80 to 59 points out of a maximum score of 120 points).

Videofluoroscopy of phonation (VFSph) recordings were performed in lateral view before and after treatment according to a standardized protocol: three trials of vocalization at comfortable pitch and loudness level with and without a digital maneuver to constrict the jejunal graft after coating the wall by swallowing high-density barium suspension $(250 \% \mathrm{w} / \mathrm{v})$. Visuoperceptual analysis was performed by two independent experienced raters blinded for therapy. The tonicity of the jejunal graft was judged 
Table 1 Tailored voice and swallowing rehabilitation program

Rehabilitation program (30 min per session, three times per week)

Therapy Effect

Tracheojejunal voice

Digital pressure on the jejunal graft at the level of the fifth cervical vertebra

Respiratory exercises (optimization of head and body positioning)

Swallow

Tongue base exercises (tongue-holding maneuver, stretch and push against resistance tasks)

Bolus modification like changing bolus volume and/or consistency (increasing bolus volumes, thin-thick liquids, pureed food)

Digital pressure at the level of the proximal (cervical) part of the jejunal graft

Postural technique consisting of extending the neck
Constriction of the jejunal graft improving vibration capacities (neoglottis)

Improvement of the airflow through jejunal constriction

Strengthening the musculature improving bolus transport into the entrance of the jejunal graft

Facilitation of bolus transport through the jejunal graft

Minimization of the jejunal convolutions by stretching the graft

Facilitation of bolus transport through the jejunal graft
Fig. 1 Pictures of the patient performing, a digital pressure maneuver during phonation, $\mathbf{b}$ postural maneuver extension of the neck during swallowing, $\mathbf{c}$ digital maneuver to stretch the jejunal graft during swallowing (arrow indicates direction of the maneuver)
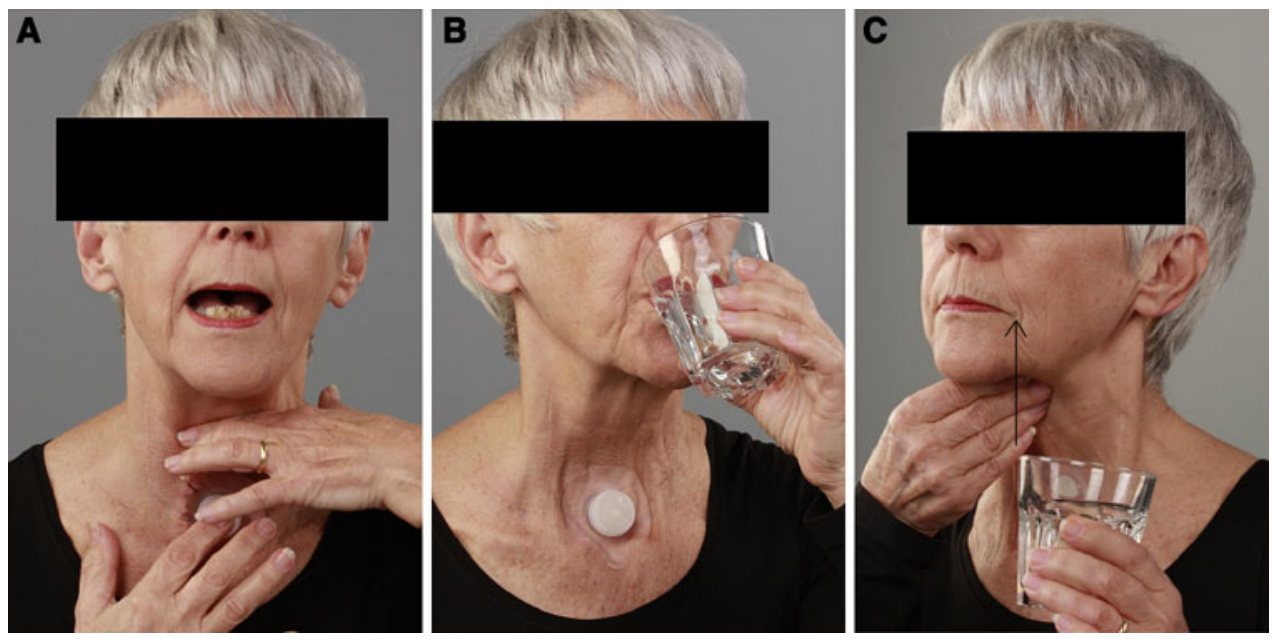

Table 2 Pre- and post-therapy measurements of tracheojejunal voice production and swallowing

\section{Tracheojejunal voice}

Esophageal insufflation test [10] (exclusively pre-therapy)

Voice Handicap Index [11]

VFSph [12]

High-speed kymograph recording [13] (exclusively post-therapy)

Swallow

FEES [14]

VFSs [15]

during phonation [12]. Before treatment, the tonicity of the jejunal wall seemed hypotonic because there was no constriction of the vibrating segment during phonation. Instead, severe dilatation of the jejunal graft was observed (Fig. 2a). Also, pooling of barium suspension at the jejunoesophageal anastomosis was seen.
After treatment, the tracheojejunal speech sounded more fluent, with increased intelligibility during digital pressure, which causes a jejunal constriction independent of intestinal peristalsis [3]. VFSph showed that the vibration capacities of the jejunal wall had improved significantly without digital pressure. The vibration capacities improved even more during the digital pressure maneuver, resulting in increased constriction and tonicity of the jejunal segment. The digital pressure could be identified at the level of the second and third cervical vertebra. The vibration capacities of the jejunal tissue improved even more during the application of digital pressure on the jejunal segment at the level of the fifth cervical vertebra (Fig. 2b). After treatment the irregular jejunal vibrations also were visualized with high-speed digital endoscopy (Fig. 3a).

Recordings were made with and without the digital pressure maneuver using a digital high-speed camera system (HreS Endocam 5562, R. Wolf, Knittlingen, Germany) in combination with a flexible nasoendoscope. The 

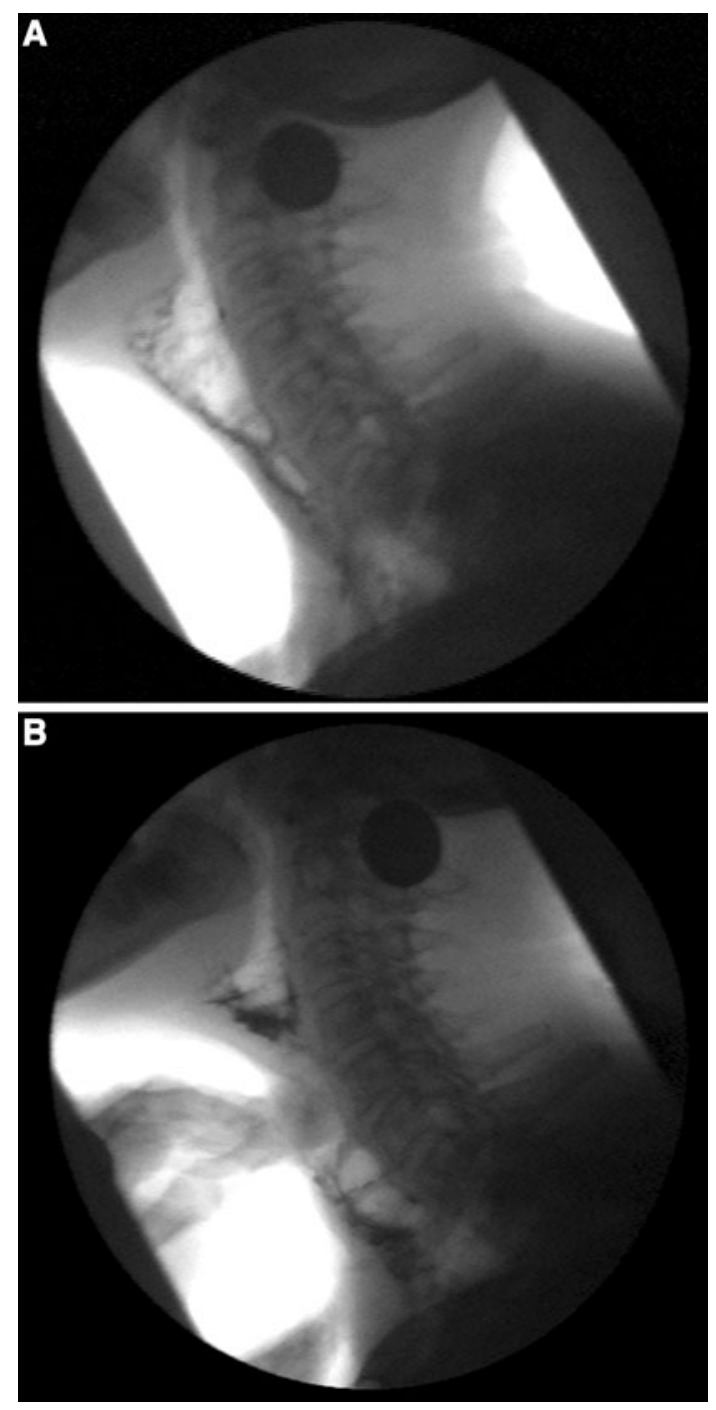

Fig. 2 VFSph. a Voicing without maneuver showed extreme ballooning of the jejunal graft during voluntary phonation. b Voicing with digital pressure maneuver at the level of the fifth vertebra showed an improvement of constriction of the vibrating jejunal segment

recording rate was 2,000 frames per second. The patient was asked to produce a number of sustained /a:/ vowels [13]. The starting point of the irregular jejunal vibration could not be identified exactly. However, the final part of the traveling vibration was situated all over the walls of the entire jejunal segment. The shape of the vibrating jejunal segment varied from a circular to an elliptical appearance. The open phase of vibration was longer than the closed phase of vibration. During digital pressure on the jejunal segment, the mucosal wave even improved (Fig. 3b). The fundamental frequency calculated from the high-speed recording was $40 \mathrm{~Hz}$ during the application of digital pressure.

\section{Swallowing}

Before and after treatment, a standardized FEES examination was performed by an experienced laryngologist [14]. Both recordings were made using a fiber-optic nasoendoscope in combination with an endoscopic digital camera (stroboview ACLS, Alphatron Medical Systems, Rotterdam, The Netherlands). Before and after therapy, the patient was offered trials of thin or thick liquid by means of a syringe. Each trial contained $10 \mathrm{ml}$ of water or apple sauce, which was dyed by methylene blue. The VFSs protocol consisted of four trials of thick liquid $(4 \times 10 \mathrm{ml}$ high-density barium boluses, $250 \% \mathrm{w} / \mathrm{v}$ ) with the patient in the laterolateral position, seated upright [15]. During preand post-therapy FEES and VFSs, several trials of liquid, as described before, were performed while applying a suprastoma-located digital maneuver in an attempt to stretch the jejunal graft. Furthermore, a postural maneuver extension of the neck was used to facilitate the act of swallowing. The oral phase before and after treatment was normal. The FEES and VFSs were judged independently by a speech-language pathologist and a laryngologist using visuoperceptual parameters: pooling in the jejunal convolutions and at the level of the jejunoesophageal anastomosis after swallowing, and jejunal reflux. During the evaluation of FEES and VFSs, the raters were blinded during assessment, before versus after therapy. During post-treatment FEES and VFSs without maneuvers, moderate symmetrical pooling in the jejunal convolutions and at the jejunoesophageal anastomosis was observed, in contrast with severe pretreatment pooling (Figs. 4a, 5a). During post-therapy measurement, the digital maneuver to stretch the jejunal graft and the postural maneuver to extend the neck did decrease pooling resulting in minor traces of bolus contrast in the jejunal convolutions and at the jejunoesophageal anastomosis after swallowing, in
Fig. 3 High-speed kymography (arrow indicates mucosal wave). a Recording of the neoglottis without digital pressure maneuver $(1,000$ frames). $\mathbf{b}$ Recording of the neoglottis with digital pressure maneuver (2,000 frames)
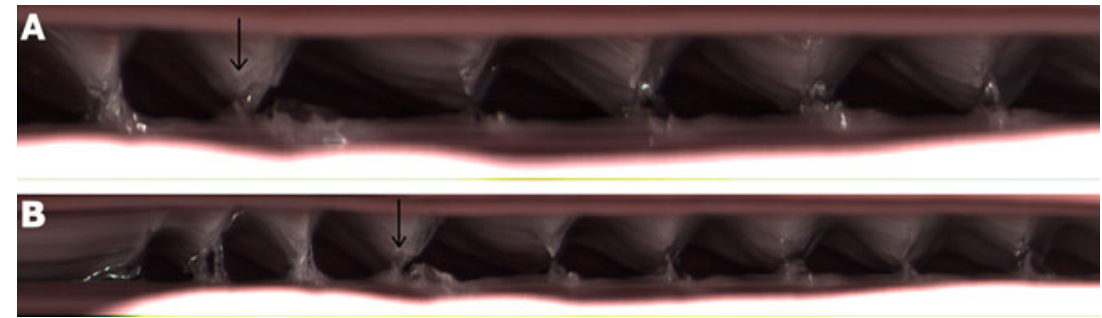
contrast with moderate to severe pre-therapy pooling using these maneuvers (Fig. 4b, c, 5b, c). Before treatment the maneuvers were less skillfully applied by the patient and thereby were less efficient with respect to postswallow pooling. Jejunal reflux was not observed after therapy in contrast with severe jejunal reflux before treatment.
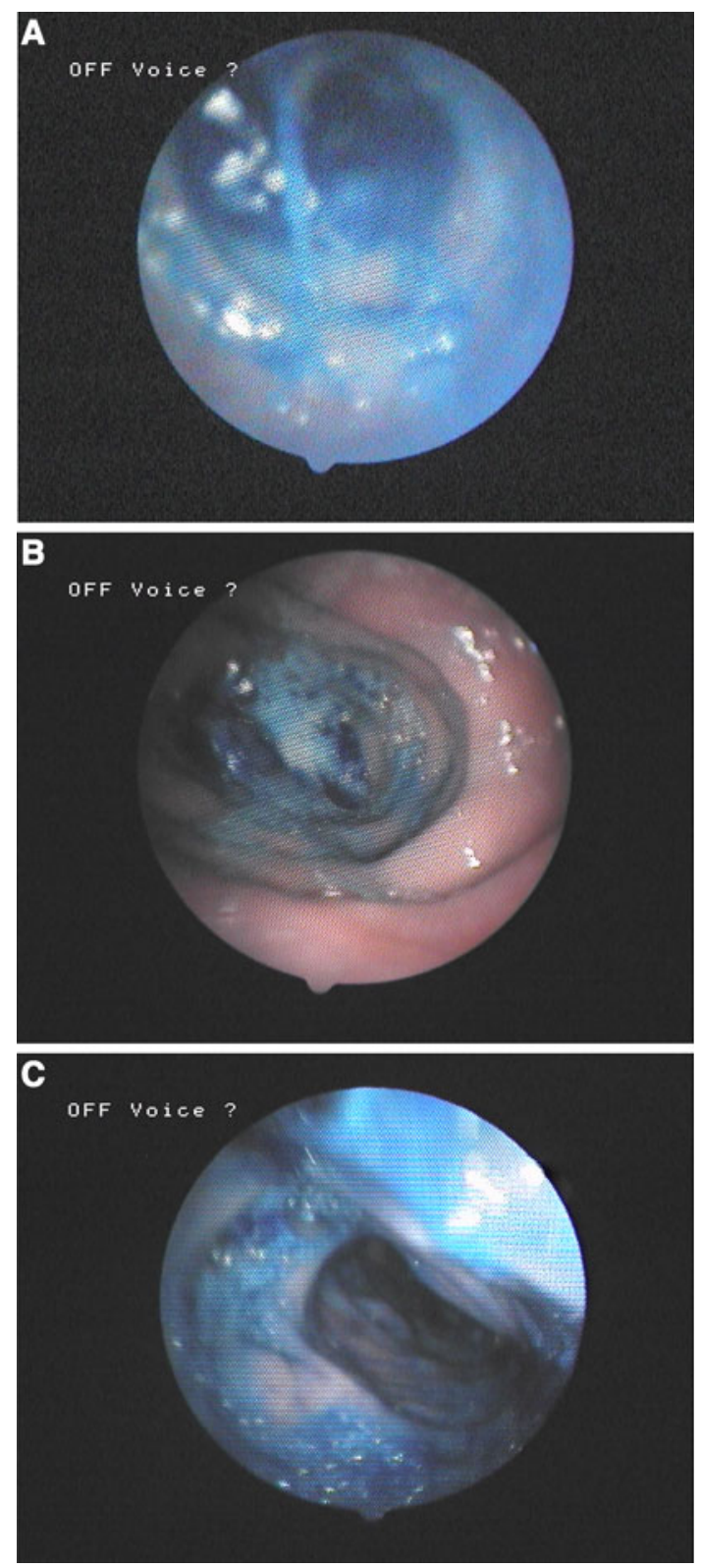

Fig. 4 FEES. a Swallowing without the digital stretching maneuver showed severe pooling in the jejunal convolutions after swallowing thick liquid (pre-therapy). b Swallowing with the digital stretching maneuver showed minor traces of bolus in the jejunal convolutions after swallowing thick liquid (post-therapy). c Swallowing with postural maneuver extension of the neck during swallowing showed minor traces of bolus in the jejunal convolutions after swallowing thick liquid (post-therapy)
Overall, the patient reported to be very satisfied with her progress in oral communication and swallowing function.

\section{Discussion}

Following circumferential pharyngolaryngectomy with free jejunal repair, the tracheojejunal voice and swallowing functions were disappointing for the patient. Initially, the prosthetic tracheojejunal voice was very weak, with extremely inadequate intelligibility. An esophageal insufflation test confirmed this finding. A second digital maneuver to constrict the graft was applied simultaneously with training of the prosthetic tracheojejunal voice. Digital pressure at a specific location of the graft prevented ballooning and induced a vibration of the wall, creating a neoglottis. The neoglottis led to an improvement in the tracheojejunal voice and thereby an improvement in the fluency and intelligibility of speech in this patient [7]. This improved tracheojejunal voice became more socially acceptable for the patient, as shown by the VHI score. VFSph identified the location of best vocal performance. The digital and postural maneuvers were gone over several times per week during a longer period of time. After several weeks of therapy the patient was able to apply these maneuvers skillfully at the exact locations of best performance. The results showed that these techniques may provide a long-term facilitation of the swallowing and speech functions in this case of prosthetic tracheojejunal voice following circumferential pharyngolaryngectomy and neopharyngeal reconstruction with a jejunal free flap. Digital high-speed endoscopy could provide useful images of the irregular vibration of the jejunal graft in prosthetic tracheojejunal voice production and may indicate the presence of a neoglottis as was observed in this patient. In the future, high-speed digital endoscopy recordings may serve as a biofeedback tool during rehabilitation of tracheojejunal voice. This information could be helpful in the multidimensional assessment and the treatment program of the tracheojejunal voice in patients following a circumferential pharyngolaryngectomy and neopharyngeal reconstruction with a jejunal free flap. In our patient, a digital maneuver to stretch the jejunal graft was applied simultaneously with dysphagia treatment by a speech-language pathologist to facilitate swallowing. Manually stretching the jejunal graft led to a decrease in convolutions which resulted in more efficient bolus transport and less pooling after swallowing, as seen during FEES and VFSs. A postural maneuver consisting of extension of the neck was taught. This postural maneuver also caused an improvement in postswallow pooling in the convolutions during FEES and VFSs, similar to the effect of digital stretching. This maneuver was even more convenient for 

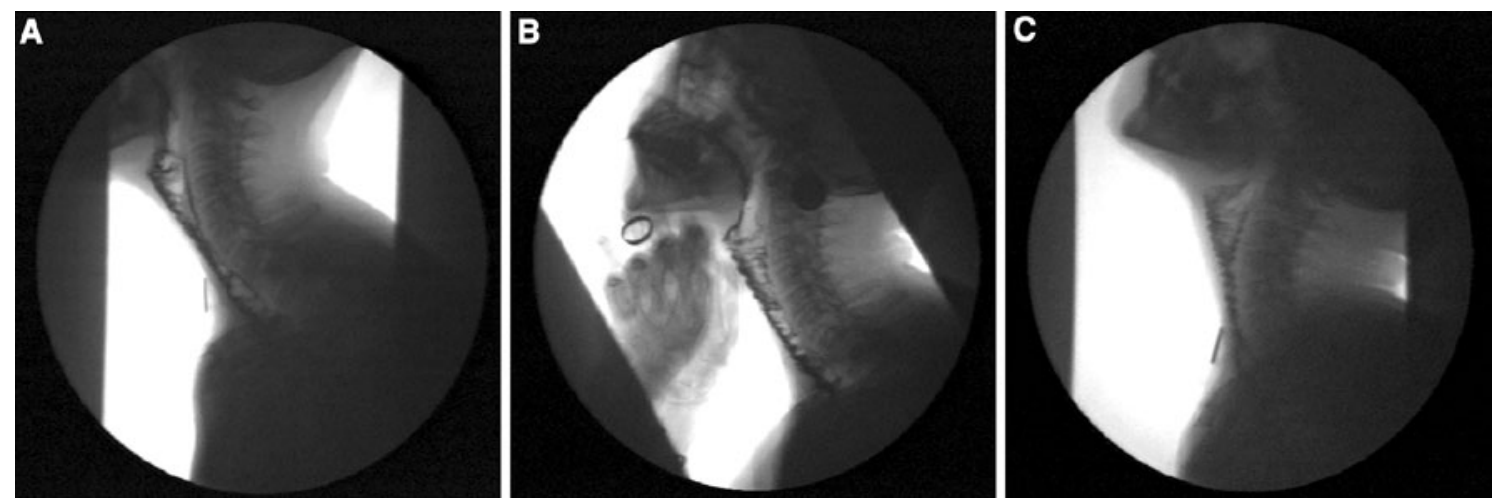

Fig. 5 VFSs. a Swallowing without maneuver showed severe pooling in the jejunal convolutions after swallowing thick liquid (pre-therapy). b Swallowing with digital stretching of the jejunal graft showed minor traces of bolus in the jejunal convolutions after

the patient because it could be performed hands free. These techniques supported the ability to increase the oral intake and increase the level of comfort during feeding.

\section{Conclusion}

There currently is no universal protocol for rehabilitation of swallowing or tracheojejunal voice function following circumferential pharyngolaryngectomy and neopharyngeal reconstruction with a jejunal free flap. Rehabilitation of prosthetic voice and swallowing function following such extensive surgery remains a challenge. This case report describes useful diagnostic assessment/imaging tools to use for performing an evidence-based evaluation of speech and swallowing functions and of the rehabilitative options in such a patient population. Positive therapy outcomes in this case seem to be mainly due to the application of digital and postural maneuvers that facilitate swallowing and the tracheojejunal voice. The offered treatment protocol in this case could be used in other motivated patients with difficulties in rehabilitation of the tracheojejunal voice and swallowing function following jejunal transfer. The application of the above-mentioned assessment/imaging tools before and after therapy is necessary in order to evaluate the effects of treatment.

Open Access This article is distributed under the terms of the Creative Commons Attribution Noncommercial License which permits any noncommercial use, distribution, and reproduction in any medium, provided the original author(s) and source are credited.

\section{References}

1. Singer MI, Blom ED. An endoscopic technique for restoration of voice after laryngectomy. Ann Otol Rhinol Laryngol. 1980;89: $529-33$. swallowing thick liquid (post-therapy). c Swallowing with postural maneuver extension of the neck during swallowing showed minor traces of bolus in the jejunal convolutions after swallowing thick liquid (post-therapy)

2. Hamaker RC, Singer MI, Blom ED, Daniels HA. Primary voice restoration at laryngectomy. Arch Otolaryngol. 1985;111: $182-6$.

3. Furuta Y, Homma A, Nagahashi T, Oridate N, Mesuda Y, Nishizawa $\mathrm{N}$, et al. Voice restoration by primary insertion of indwelling voice prosthesis following circumferential pharyngolaryngectomy with free jejunal graft. Auris Nasus Larynx. 2005;32:269-74.

4. Wilson P, Bruce-Lockhart F, Johnson A, Rhys Evans P. Speech restoration following total laryngo-pharyngectomy with free jejunal repair. Clin Otolaryngol. 1994;19:145-8.

5. Ward E, Bishop B, Frisby J, Stevens M. Swallowing outcomes following laryngectomy and pharyngectomy. Arch Otolaryngol Head Neck Surg. 2002;128:181-6.

6. Hilgers FJM, Hoorweg JJ, Kroon BBR, Schaeffer B, de Boer JB, Balm AJM. Prosthetic voice rehabilitation with the Provox system after extensive pharyngeal resection and reconstruction. In: Algaba J, editors. Surgery and prosthetic voice restoration after total and subtotal laryngectomy. Proceedings of the 6th international congress on surgical and prosthetic voice restoration after total laryngectomy, San Sebastian, 29 September-1 October 1995. Excerpta Medica International Congress Series 1112. Amsterdam: Elsevier; 1996, p. 111-120.

7. Bleach N, Peery A, Cheesman A. Surgical voice restoration with the Blom-Singer prosthesis following laryngopharyngoesophagectomy and pharyngogastric anastomosis. Ann Otol Rhinol Laryngol. 1991;100:142-7.

8. Crary MA, Groher ME. Overview of behavioral treatment strategies. In: Introduction to adult swallowing disorders. St. Louis: Butterworth Heinemann; 2003, p. 179-198.

9. Langmore S, Miller RM. Behavioral treatment for adults with oropharyngeal dysphagia. Arch Phys Med Rehabil. 1994;75: 1154-9.

10. Blom E, Singer M, Hamaker R. An improved esophageal insufflation test. Arch Otolaryngol. 1985;111:211-2.

11. Jacobson BH, Johnson A, Grywalski C. The Voice Handicap Index (VHI): development and validation. Am J Speech Lang Pathol. 1997;6:66-70.

12. van As C, Op de Coul B, van den Hoogen F, Koopmans-van Beinum F, Hilgers F. Quantitative videofluoroscopy. Arch Otolaryngol Head Neck Surg. 2001;127:161-9.

13. van As C, Tiggers M, Wittenberg T, Op de Coul B, Eysholdt U, Hilgers F. High-speed digital imaging of neoglottic vibration after total laryngectomy. Arch Otolaryngol Head Neck Surg. 1999;125:891-7. 
14. Langmore SE, Aviv JE. Endoscopic procedures to evaluate oropharyngeal swallowing. In: Endoscopic evaluation and treatment of swallowing disorders. New York: Thieme; 2001, p. $73-100$.

15. Logemann JA. Manual for the videofluorographic study of swallowing. 2nd ed. Austin, TX: Pro-Ed; 1998.
Laura W. J. Baijens MD

Renée Speyer MS, SLP, PhD

Nel Roodenburg SLP

Frans J. M. Hilgers $\mathrm{MD}, \mathrm{PhD}$ 